\title{
A Telehealth System for Remote Auditory Evoked Potential Monitoring
}

\author{
Jorge Millan MsC, PhD; Member, IEEE ${ }^{1}$, and Leonardo Yunda, MsE, \\ $\mathrm{MsC}, \mathrm{PhD}(\mathrm{c})$, Member, $\mathrm{IEEE}^{2}$
}

\author{
${ }^{1}$ Jorge Millan is Executive Director from Hialeha Technology Center, HIATEC and Science \\ Director from Sigma Biomedical, Hialeah, FL 33010, USA. \\ ${ }^{2}$ Leonardo Yunda is Dean School of Health Sciences from UNAD and work with BIOINNOVA \\ Group Research from UNAD, Bogotá D.C., Colombia.
}

Correspondence: jmillan@sigmabiomedical.com, leyunda@ieee.org

Recibido: 23/03/2013 Aceptado: 14/06/2013

\begin{abstract}
A portable, Internet-based EEG/Auditory Evoked Potential (AEP) monitoring system was developed for remote electrophysiological studies during sleep. The system records EEG/AEP simultaneously at the subject's home for increased comfort and flexibility. The system provides simultaneous recording and remote viewing of EEG, EMG and EOG waves and allows on-line averaging of auditory evoked potentials. The design allows the recording of all major AEP components (brainstem, middle and late latency EPs) and constituent single sweep EEG epochs. Auditory stimulation is remotely controlled from any internet-connected PC. The developed platform consists of a portable server computer for data acquisition and secured data transmission to the Internet, a web server for temporary storage, and a remote viewing station. During operation, signals are acquired during sleep and transmitted to the secured web server for controlled access viewing. The remote viewer station allows the user to continuously visualize the auditory evoked potentials, modify auditory stimuli at any time and adjust system parameters for its online computation. The developed internet-based EEG/EP system has been tested in a laboratory environment. Preliminary results demonstrate feasibility of remote real-time acquisition and viewing of EEG and EPs and future potential for home monitoring and telemedicine applications.
\end{abstract}

Keywords: auditory evoked potentials, remote monitoring, telemedicine, EEG. 


\section{Introduction}

Evoked potentials (EP's) are electrical signals originated in the brain in response to an external sensory stimulus. Fast onset auditory stimuli such as clicks and tone bursts, elicit Auditory Evoked Potentials (AEP's) within structures of the cochlea, auditory nerve and auditory pathways in the brain. AEP signals, embedded in the ongoing electroencephalogram (EEG) waves, are extracted by the averaging of a synchronized set of post stimulus EEG sweeps. For clinical diagnosis, the extracted waveform is further decomposed in time into Auditory Brainstem Response (ABR), Middle Latency Response (MLR) and Late Latency Response (LLR) components, each corresponding to a time window of the AEP response with respect to the stimulus onset. Each of these responses has its origin in a specific section of the brain and reveals specific neurological conditions, states and pathologies. Widespread clinical use of EP methodology has made the functional testing of many sensory and motor neural pathways possible in both normal and disease states.

Monitoring of auditory evoked potentials takes long to perform due to poor signal-to-noise (SNR) ratios, which impose the need to average several hundred EEG sweeps to obtain reliable responses. Monitoring of AEP can extend up to several hours within which the subject is usually asleep. In applications that require long term monitoring, AEP monitoring at the subject's home or natural sleep environment have the potential to reduce the disturbing uncomfortable factors that are present in a sleep lab, thus improving the clinical setting for monitoring studies. Due to its safe non-invasive principle, AEP studies could then be performed remotely through a telemedicine application reducing operating costs and increasing patient comfort and study flexibility.

Several groups throughout the years have reported the implementation of telemedicine applications for remote neurophysiological monitoring. EEG data transmission over telephone links (1) and high-speed digital networks (2) has been developed and implemented for real time monitoring as well as for routine offline EEG review and interpretation (3). Connectivity schemes include data transmission from homes as well as from intermediate clinical centers to reference centers for expert review. Recent implementations include transmission of EEG data through the Internet via web applications (4), as well as the implementation of software packages and computer information systems (5-9) for remote data collection, review, analysis and teleconsultation. Some advanced developments have included video consultation (2), EEG review and analysis environments based on web (6-7) and virtual reality technologies (8), and the implementation of remote patient supervision in the use of a brain-computer interface system (9). Clinical applications include standard electroencephalography (5), epilepsy (5), (10-11), (16), sleep medicine (1,12-15), as well as applications for emergency and screening purposes (7),(16).

Although remote EEG monitoring has been successfully implemented and well documented, demonstrating the benefits and feasibility of continuous operation in various clinical settings (3), $(17,18)$, there seems to be no published reports on remote auditory evoked potential recording and processing. Though an Automated Visual Evoked Potential (VEP) system (19) was implemented to collect, analyze and off-line transmit via a telephone link VEP averaged data to an evaluation center from the acquisition lab, this system was mainly designed to standardize the acquisition of data for visual evoked potential analysis between different labs. The system was not intended for remote home monitoring, and did not seem to possess real time EEG data capture and transmission capabilities.

Due to the growing importance of evoked potential studies for neurological assessment and the clear advantages of remote EEG monitoring, the aim of the present work was to design and construct an auditory evoked potential monitoring 
system to remotely monitor the subject's sleep condition while providing capabilities to remotely elicit auditory stimulus during the various sleep stages. The remote monitoring system is intended to receive and online display EEG, electromyogram (EMG) and electrooculogram (EOG) waves from any Internet connected PC through an authorized secure connection and provide tools to calculate display and process the extracted evoked auditory potentials.

\section{A. System Overview}

The portable AEP monitoring system has been developed around a Windows XP laptop computer, a PCMCIA data acquisition card, a bioelectric amplifier system and an Internet web server. The software application is designed to acquire, manage and transfer electrophysiological data such as EEG, EMG and EOG to the Internet web server; generate and provide auditory stimulus control, and respond to remote operator requests delivered by a web server connection. The system allows multiple authorized users in remote locations to simultaneously access physiological data and compute and visualize averaged evoked responses. Continuous viewing of EEG, EMG and EOG signals allows the operator to determine the subject's sleep stage. In addition, transmission of EEG data sweeps allow the implementation of selective averaging and artifact rejection filters in the computation of evoked potentials at the remote site.

The software platform is comprised of the following application modules: 1) data acquisition and storage; 2) stimulus control; 3) stimulus generation; 4) communications server; 5) communications client; 6) Internet web server; 7) EEG and AEP viewer; 8) AEP processing; 9) messaging system; and 10) system control.

These modules are functionally connected as shown in Fig. 1. The system consists of the local machine, the Internet web server and the remote machine. The local machine includes the home
PC, bioamplifiers, data acquisition and software modules for data acquisition and storage, stimulus control, data communication, messaging system and system control. The remote machine includes the remote PC running modules for Internet communication, physiological signal viewer, AEP processing and stimulus generation. The software modules run as independent applications, which are initiated, launched and continuously monitored by the system control module. This module detects component lockouts, especially when momentarily losing Internet connection, and responds by terminating and initiating a new instance of the unresponsive module. Synchronization and sequential data information is continuously maintained. The messaging system enables twoway communication between the different modules, necessary to transmit requests and status information.

The developed platform is modular and easily upgradeable. System maintenance and upgradeability is readily obtained due to the modular nature of the system. Faster and more efficient components can be easily integrated into the system in later software revisions.

\section{B. Analog Front End}

Electrophysiological signals are brought to a Grass Telefactor (West Warwick, RI) model 12 neurodata acquisition system. Electrical signals are bandpass filtered (0.3 - up to $3000 \mathrm{~Hz}, 6 \mathrm{~dB} /$ Octave) and amplified with a gain of up to 50,000. Once bioelectrical signals are filtered and amplified, they are digitized by a National Instruments (Austin, TX) data acquisition card model DAQCard-1200. The DAQCard-1200 card is a 12-bit analog I/O card with 8 analog input channels, 2 analog output channels and 24 digital Input/Output lines. The maximum sampling rate is $100 \mathrm{KHz}$ for single channel acquisition. The DAQCard-1200 is fully software configurable. This capability is exploited to generate stimulus signals such as clicks and tone bursts of arbitrary shape, duration and repetition rate. In order to locate the stimulus onset along 
the acquired EEG traces, the stimulus signal is then continuously digitized with the same time base as the EEG signals. The stimulus occurrences are then detected and time stamped in all EEG traces for later use in the averaging routines.

\section{Signal Processing}

To minimize buffer size and maximize data throughput prior to transmission, down sampling is performed on the low frequency EMG and EOG signals. To obtain the ABR components from the post stimulus EEG epochs, the data acquisition is setup at the highest possible sampling rate that does not cause cumulative delays in the receiving site. The electrical pulse generating the auditory stimulus is also sampled at the highest possible rate to increase time localization of the stimulus onset in the EEG sweep, which minimizes jitter in the averaging process. In operation all acquired electrical signals are digitized at $10 \mathrm{KHz}$. Digitized EMG and EOG signals are next down sampled at a rate of 20:1, for an effective sampling rate of 500 $\mathrm{Hz}$. To further reduce buffer size, the stimulus onset is obtained from the digitized stimulus signal by a peak detector and its position along the EEG trace is transmitted instead. The overall reduction process is completed within the time to acquire the next chunk of data.

\section{Stimulus Generation}

The stimulus generation scheme is based on online requests by the remote operator, monitored and executed by the data acquisition system. The requirement of the generation scheme is the ability to remotely elicit any stimulus at any time during the duration of the study. Since the system is to be used during sleep, different stimuli can be setup for different sleep stages. Intensity levels can be modified according with the hearing threshold at the particular sleep stage. The stimulus sequence can also be started once the subject is asleep, thus providing comfort and an ideal environment to the subject.

Auditory stimulus is generated from the DAQCard-1200 card and delivered to the subject via a Cabot Safety Corporation (Southbridge, MA) insert earphone model EARTONE 3A. The stimulus is software controlled by a module that synthesizes predefined and user defined waveforms, which are sent to the DAQCard-1200 for analog output. In a study session, the local stimulus control module routinely monitors the Internet web server for changes in auditory stimulus settings such as intensity, rate and waveform that may have been requested by the remote operator. Requests for start/stop of the stimulus occurrence are also monitored and the corresponding action is performed. The operator has complete remote control on the stimulus generation, which may as well be set to run in stand-alone mode.

In the remote operators' side, a stimulus generation module allows the user to synthesize arbitrary stimulus waveforms and specify rate and intensity levels while the study is being performed. The synthesized waveforms are graphically displayed to the user for visual confirmation. Once the user has defined the stimulus waveform, the client module uploads it to the web server where it will be accessed by the stimulus control module of the local server PC. The total time it takes from uploading the stimulus settings to the server, to the observation of a corresponding EEG response in the operators' side is around 3 seconds.

\section{E. Data Transmission and Reception}

Two independent software modules, the server and the client, are in charge of bi-directional data transfer operations to and from the Internet server. The server module at the local machine continuously monitors the occurrence of new data written to the hard disk by the data acquisition module. Once new data corresponding to one second of acquisition is acquired, an appropriate header, containing synchronization, error checking and identification information, is added to the data packet before it is transmitted. The server then uploads the data to the Internet server via the FTP protocol. The data is saved in the web 
server in a data file until a new data packet comes and rewrites its contents. The server module also has the ability to send particular data files to the Internet sever upon request by the client module in case that a data file is lost.

The client module located at the remote machine continuously checks for the occurrence of new information in the web server. The module access and downloads the current data file via http commands, checks for its integrity and determines if the data corresponds to a new packet or to an old one. If the data corresponds to new data then if saves it into the system for online display and analysis. In case the data file corresponds to a previously saved file, it ignores it and continuous the monitoring process. If the data has been rewritten before the client accesses it or in case of data is lost, the client module then sends a request that the particular data packet be uploaded again to the Internet from the server PC.

\section{F. Auditory Evoked Potentials Analysis}

The downloaded data files containing EEG, EMG and EOG signals as well as stimulus synchronization data are loaded and separated accordingly for real time analysis. Stimulus onset positions are used to split the EEG trace into segments of fixed duration, with each segment being of less duration than the inter stimulus interval. Split EEG sweeps are then added to form the averaged evoked potential. Averages can consist of 512, 1024 or any user defined number of post stimulus EEG sweeps. An artifact rejection filter eliminates from the averaging buffer EEG signals outside a predefined voltage range, which indicate artifacts due to motion or to an external noise source. The program allows viewing the resulting running averaging as new EEG sweeps are added to the averaging process. The EAP analysis module also allows online display of EEG, EMG and EOG, and offline viewing of AEPs from previous acquisitions. This analysis scheme allows detecting errors or possible problems during the study.

\section{G. Data Security}

Network security aspects are managed by the web server. A secure server connection is established and authorized users are required to enter the assigned security information during the login session. As a secure measure, patient personal information is not encoded into the transmitted data packet, since only the system ID needs be transmitted.

\section{H. Graphical User Interface}

The graphical user interface (GUI) is windows menu and icon driven, and allows the remote operator to easily select and set auditory stimulus parameters, and manipulate the graphics display to adjust voltage and time scales in the presentation of the electrophysiological signals. The GUI also allows viewing and graphic manipulation of the auditory evoked potentials waveforms, as well as to set parameters for their computation such as amplitude rejection filters or selective addition/deletion of EEG sweeps (selective averaging).

\section{Verification and Validation}

Each modular component of the system was independently tested, verified and validated by appropriate software and hardware test modules. Test routines checking data integrity in the received data files were run. Local and remote stimulus generation was verified using a digitizing oscilloscope, with signals of various shapes and intensities. The averaging routines were tested and validated by PC-generated electrical signals of known shapes. The system integration was also tested and showed to perform according with requirements and specifications. The recorded AEPs were in good correspondence in terms of shape and latencies to the ones obtained from the SmartEP AEP system.

\section{Results}

Laboratory in-house tests were performed to obtain first insights in the operation of the remote monitoring system. Lab personnel volunteered to participate in the system tests. Electrode montages included EEG (2) at +forehead, -ipsi mastoid, and 
ground contra mastoid, EOG (2) and EMG (2) at chin. The volunteer subject was asked to set relax in a supine position with the eyes closed. Auditory monaural rarefaction clicks were elicited at $60-\mathrm{dB}$ $\mathrm{nHL}$, with a repetition rate of $4 \mathrm{~Hz}$. Bandpass filter settings were $0.3-3000 \mathrm{~Hz}$ for EEG, 0.1-100 $\mathrm{Hz}$ for EMG, and 1-100 $\mathrm{Hz}$ for EOG, all at the amplifier gain of 50,000. The DAQCard-1200 was set to a sampling frequency of $9.6 \mathrm{KHz}$, with 20 microseconds time resolution for the stimulus waveform generation. The Internet connection speed was more than $128 \mathrm{~KB}$ s for file upload and $256 \mathrm{~KB}$ s for file download. In operation, the complete processed data buffer is 23 Kbytes in size, and it takes around 200 milliseconds to transmit to the Internet web server. From there, it then takes around 100 milliseconds to download to the remote PC viewer. The total latency to view the electrophysiological signals at the remote machine is around 2 seconds, from the time they are acquired at the local machine. The total time for the auditory stimulus to be elicited once the operator adjusts the parameters is around 2 seconds.

Fig. 1 illustrates the GUI for the remote operator stimulus control, where an elicited tone burst is shown. Sliders are used to setup the tone frequen$c y$, intensity and duration. Prerecorded tones can also be loaded and sent to the subject. Fig. 2 and Fig. 3 illustrate laboratory results obtained with the remote monitoring system. EEG/EMG and EOG traces corresponding to 1 second of acquisition are displayed in Fig. 2 as well as the AEP signals after the averaging process is completed. The stimulus signals are also displayed on the screen (lower trace). As new EEG epochs are received at the remote site, the AEP is continuously recalculated, updated and displayed on the operators screen. Fig. 3 (left) shows ABR, MLR and LLR components from the average of 5 consecutive runs within the hour. Fig. 3 (right) illustrates AEPs from three consecutive days from the same subject. Good system performance is observed. All transmitted signals were stored in the local as well as in the remote machine for further data integrity checks.

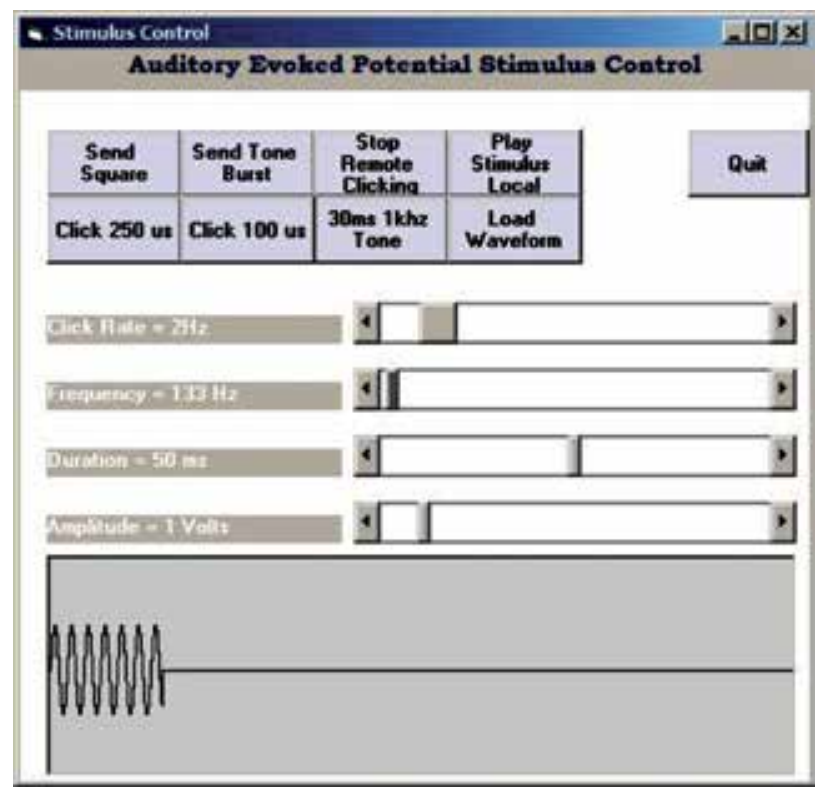

Figura 1. Client GUI for remote stimulus generation. Clicks, tone bursts or arbitrary waveforms can be synthesized or loaded, displayed and sent to the server's stimulus control module.

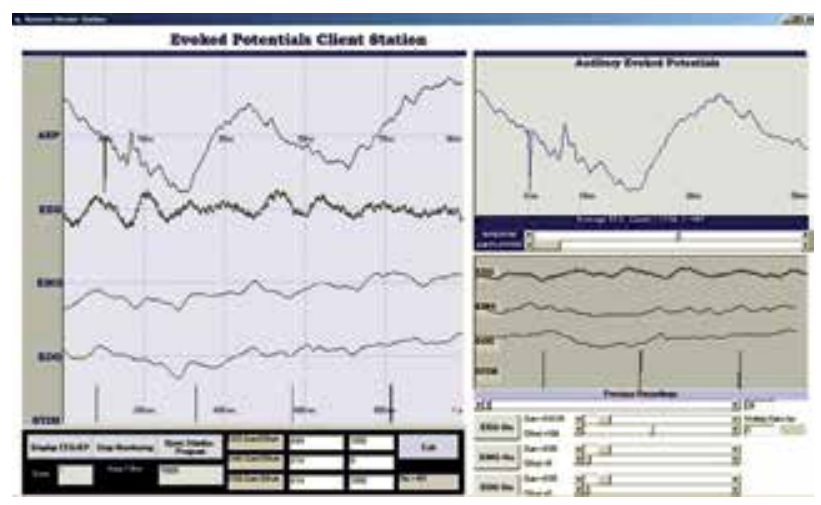

Figura 2. Client station GUI illustrating EEG/EMG/EOG as well as click onset signals and running average to calculate the auditory evoked potential signal. A secondary window displays previously saved recordings for reference during analysis.
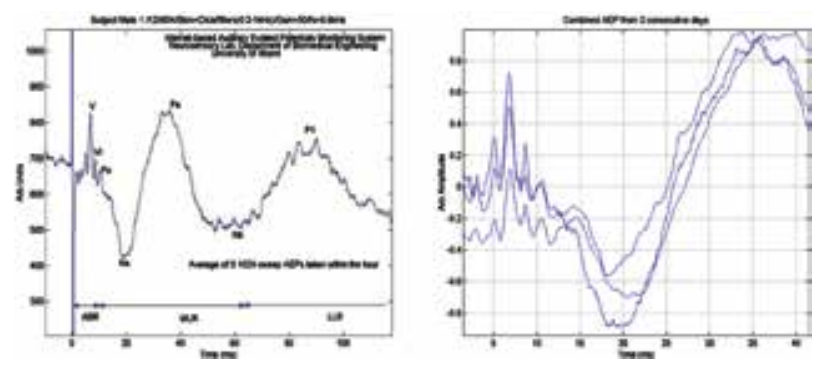

Figura 3. Preliminary results demonstrating averaged auditory evoked potentials. Left figure shows the average of 5 consecutive 1024 EEG sweeps AEPs obtained within the hour. ABR, MLR and LLR. 


\section{Discussion}

Remote monitoring of EEG and auditory evoked potential signals was successfully implemented. The system architecture is modular and easily upgradeable. The developed system is portable and easily configurable and expandable to include remote monitoring of various physiological variables for various telemedicine applications and clinical scenarios. The data acquisition is easily programmable even during operation, allowing resetting the sampling rate according to the bandwidth of the internet connection. An option exists to continuously calculate and transmit the auditory evoked potential from the server side, reducing this way the required transmission bandwidth. In this scheme, continuously transmitting EEG/EMG/EOG and processed AEP will require less than 28 kilobits per second, which is obtained using standard phone lines. Future additional features to include are a video camera to monitor the subject's sleep condition, and an alarm to alert the subject of any contact problems that may be occurring with the physiological channels during acquisition.

An interesting feature compared to several available auditory evoked potential systems is the ability of the developed system to capture record and display raw EEG/EEG/EMG waves, which are needed in the screening of the sleep stages or for off-line analysis and recalculation of evoked potentials, using different filter settings or implementing selective averaging methods. The ability to remotely control the auditory stimulus allows the operator to select the stimulus type, intensity, duration and the time window in the sleep stage during which the stimulus remains active. This stimulus control scheme is ideal for studies relating the pre stimulus EEG frequency during various sleep stages and the auditory evoked potential response.

The developed system allows non specialized hospitals or health centers in remote areas to provide and conduct auditory evoked potential sessions and have the remote expert provide the expert analysis through an Internet connection. Applications of the current system include remote expert consulting, remote neurological assessment, mobile emergency medicine, home monitoring of auditory evoked potentials in children to asses hearing thresholds during deep sleep, as well as for analysis of auditory evoked responses during the different sleep stages.

\section{Conclusion}

We have developed an Internet-based EEG/Auditory Evoked Potential monitoring system. The prototype system has been tested in a laboratory environment. Preliminary results indicate equivalent performance to standard auditory evoked potential systems. The system demonstrates promising features such as remote auditory control and Internet based evoked potential analysis and monitoring. The system also shows feasibility of continuous auditory evoked potential monitoring at home during sleep. Future work may include increasing number of channels for sleep studies, and additional channels for monitoring of additional physiological variables. A future implementation also includes a real-time video camera and an alarm system to detect and alert the operator of disconnected physiological channels.

\section{References}

1. M. Rosekind, A. B. Thomas, J. Coates and C. Thoresen, “Telephone transmission of all-night polysomnographic data from subjects' homes", The Journal of Nervous and Mental Disease. 1978; 166: 438-441.

2. P Loula, E. Rauhala, M. Erkinjuntti, E. Raty, K. Hirvonen and V. Hakkinen, "Distributed clinical neurophysiology", Journal of Telemedicine and Telecare. 1997; 3: 89-95

3. Ltc. D. Kristo, A. H. Eliasson, R. Poropatich, C. M. Netzer, J. Bradley, D. Loube and N. C. Netzer, "Telemedicine in the sleep laboratory: feasibility and economic advantages of polysomnograms transferred online", Telemedicine Journal and e-Health. 2001;7(3):219-224.

4. L. De Luca, "TeleEEG: A telemedical software package for EEG”, Future Generation of Computer Systems. 1998; 14: 61-66.

5. N.B. Finnerup, A. Fuglsang-Frederiksen, P. Rossel and P. Jennum, "A computer-based information system for epilepsy and electroencephalography", International Journal of Medical Informatics. 1999; 55:127-134. 
6. M. Kuntalp and O. Akar, "A simple and low-cost Internet-based teleconsultation system that could effectively solve the health care problems in underserved areas of developing countries", Computer Methods and Programs in Biomedicine. 2004; 75: 117-126.

7. V. I. Nenov, F. Buxey and Y. Yamaguchi, "BRAVO/TeleTrend: A Comprehensive WWW-based Neuromonitoring System for the Neurosurgery ICU", Medicine Meets Virtual Reality, J. D. Westwood et. al. (Eds) IOS Press. 1999: 228-234.

8. E Jovanov, D. Starcevic, A. Samardzic, A. Marsh and Z. Obrenovic, "EEG analysis in a telemedical virtual world", Future Generation Computer Systems. 1999;15: 255-263.

9. G. Muller, C. Neuper and G. Pfurtscheller, "Implementation of a telemonitoring system for the control of an EEG-based brain-computer interface", IEEE Transactions on Neural Systems and Rehabilitation Engineering. 2003; 11: 54-59.

10. C. E. Elger and W Burr, "Advances in telecommunications concerning epilepsy”, Epilepsia. 2000; 41(5): S9-S12.

11. D. Holter, J. Cameron and C. Binnie, "Tele-EEG in epilepsy: review and initial experience with software to enable EEG review over a telephone link", Seizure. 2003; 12: 85-91.

12. F Abdenbi, A. Ahnaou, S. Royant-Parola, H. Nedelcoux, S. Rouault, D. Alfandary, C. Lepajolec and P. Escourrou, "Ambulatory sleep recording in a healthcare network: a feasibility study", C R Biologies. 2002; 325: 401-405.
13. D. Padeken, D. Sotiriou, K. Boddy and R. Gerzer, "Health Care in Remote Areas", Journal of Medical Systems. 1995; 19(1): 69-76.

14. D. Kristo, A. H. Eliasson, N. C. Netzer and T. Bigott, "Application of Telemedicine to Sleep Medicine", Sleep and Breathing. 2001; 5(1): 97-99.

15. T. Penzel, "Prototypes and Applications in Neurotelemedicine", European Neurological Network, T. Paiva and T. Penzel (Eds) IOS Press 2000: 89-99.

16. S B Uldal, J. Amerkhanov, S. Manankova, A. Mokeev and J. Norum, "A mobile telemedicine unit for emergency and screening purposes: experience from north-west Russia", Journal of Telemedicine and Telecar. 2004; 10:11-15.

17. P. Escourrou, S. Luriau, M. Rehel, H. Nedelcoux and J-L. Lanoe, "Needs and Costs of Sleep Monitoring", European Neurological Network, T. Paiva and T. Penzel (Eds) IOS Press. 2000:69-84.

18. N. Pelletier-Fleury, F. Gagnadoux, R. Rakotonanahary, J-L. Lanoe and B. Fleury, "A Cost-Minimization Study of Telemedicine", International Journal of Technology Assessment in Health Care.2001; 17(4):604-611.

19. D. Papakostopoulos, J. C. Dean Hart, S. Papakostopoulos, K. Dodson and A. Williams, "Standardized visual evoked potentials for telematic electrodiagnosis from five laboratories in three European countries", Journal of Telemedicine and Telecare. 1999; 5: 23-31. 